\title{
Clinical significance of mitofusin-2 and its signaling pathways in hepatocellular carcinoma
}

\author{
Yingsheng $\mathrm{Wu}^{1,2,3 \dagger}$, Dongkai Zhou ${ }^{1,2,3 \dagger}$, Xiaobo Xu ${ }^{1,2,3}$, Xinyi Zhao ${ }^{2,3}$, Pengfei Huang ${ }^{2,3}$, Xiaohu Zhou ${ }^{2,3}$, \\ Wei Song ${ }^{2,3}$, Hua Guo ${ }^{1,2,3}$, Weilin Wang ${ }^{1,2,3^{*}}$ and Shusen Zheng ${ }^{1,2,3^{*}}$
}

\begin{abstract}
Background: The mitochondrial GTPase mitofusin-2 (MFN2) gene encodes a mitochondrial membrane protein that can induce apoptosis of hepatocellular carcinoma (HCC) via the mitochondrial apoptotic pathway, as validated in our previous research. However, little is known of the clinical significance of MFN2 expression and its signaling pathways in HCC.

Methods: MFN2 mRNA expression in tumor and adjacent non-tumor tissues from 115 patients with HCC was investigated using quantitative real-time PCR. The association of the MFN2 mRNA expression level with clinical and pathological parameters was evaluated statistically, while a comparative microarray analysis was used to identify MFN2 signaling pathways in HepG2 cells.

Results: MFN2 was significantly $(p<0.0001)$ downregulated in HCC tissues. Low MFN2 expression was significantly correlated with sex and preoperative alpha-fetoprotein $(p<0.05)$. Both a Kaplan-Meier survival curve and multivariate analyses showed that MFN2 was related to overall survival. A comparative gene expression microarray revealed 211 upregulated (58\%) and 153 downregulated (42\%) genes. Eighteen pathways were identified as the most significant pathways correlated with MFN2.

Conclusions: Low MFN2 expression in HCC indicated a worse overall survival. Crucial signaling molecules such as PI3K-AKT, cytokine receptor, and focal adhesion may participate in MFN2-mediated signaling pathway changes in HCC.
\end{abstract}

Keywords: MFN2, Hepatocellular carcinoma, Overall survival, Network function

\section{Background}

Hepatocellular carcinoma $(\mathrm{HCC})$ is one of the most lethal cancers [1], accounting for approximately 600,000 deaths annually worldwide [2]. Viral infections, excessive alcohol intake, and exposure to aflatoxin are common risk factors for developing HCC. In China and Africa, hepatitis B virus causes more than $50 \%$ of HCC, whereas hepatitis $C$ virus is the leading cause in Europe and North America [3]. In addition, hereditary liver diseases and non-alcoholic fatty liver disease are associated with HCC. The pathogenesis of

\footnotetext{
* Correspondence: wam@zju.edu.cn; shusenzheng@zju.edu.cn

${ }^{\dagger}$ Equal contributors

${ }^{1}$ Division of Hepatobiliary and Pancreatic Surgery, Department of Surgery,

First Affiliated Hospital, School of Medicine, Zhejiang University, No. 79

Qingchun Road, Hangzhou 310003, China

Full list of author information is available at the end of the article
}

HCC includes the stepwise development of liver injury, regeneration, fibrosis and cirrhosis, dysplasia, and malignancy, which ultimately transforms normal liver cells into cancer cells via genetic and epigenetic alterations [4]. To comprehend the molecular biology of HCC, recent wholegenome research and exome sequencing analyses have found key pathway changes in HCC, including inactivation of the p53 pathway and activation of the wnt $/ \beta$-catenin and Ras/PI3K pathways and telomerase [5].

The mitofusin 2 (MFN2) gene, also known as the hyperplasia suppressor gene, encodes a protein belonging to the GTPase family that is located on the mitochondrial outer membrane $[6,7]$ and is involved not only in mitochondrial fusion but also in mitochondrial trafficking and mitophagy [8]. Mutations or 
abnormalities of MFN2 may occur in various diseases, including Charcot-Marie-Tooth disease, obesity, and diabetes mellitus [9-11]. Recent studies have shown that MFN2 overexpression suppresses the proliferation of vascular smooth muscle cells and cardiac myocytes in the rat $[12,13]$. Our research team has reported on the pro-apoptosis effects of MFN2 in HCC over the past 5 years [14-16]. Furthermore, MFN2 acts as a tumor suppressor in diverse cancers of the bladder, stomach, and lung [17-19].

However, the relationship between MFN2 expression and the clinical characteristics of HCC has not been explored. Therefore, we investigated MFN2 mRNA expression in tumor and adjacent non-tumor tissues from 115 patients with HCC and statistically evaluated the association of MFN2 mRNA expression with clinical and pathological parameters. A gene expression microarray was used to determine whether MFN2 correlated with differentially expressed genes (DEGs) in HepG2 cells. We also constructed an MFN2-related functional interaction (FI) network by mapping these DEGs to the FI data.

\section{Methods}

\section{Patients}

This study enrolled 115 patients with HCC who underwent curative hepatectomy. The study was approved by the ethics committee of the First Affiliated Hospital of Zhejiang University, and informed consent was obtained from all patients. HCC was diagnosed in all patients before or after hepatectomy and was confirmed histopathologically. None of the patients received presurgical chemotherapy or radiation therapy. The baseline characteristics of the patients are summarized in Additional file 1: Table S1. Most patients were followed regularly as outpatients. A diagnosis of recurrence was based on typical contrast computed tomography $(\mathrm{CT})$ or magnetic resonance imaging (MRI) findings.

\section{Cell lines and cell culture}

The HepG2 HCC cell line was cultured in Dulbecco's modified Eagle's medium (DMEM) (Gibco, Grand Island, NY, USA) supplemented with $10 \%$ heatinactivated fetal bovine serum (Sigma-Aldrich, St. Louis, MO, USA) and $100 \mathrm{U} / \mathrm{mL}$ penicillin/streptomycin. HepG2 cells were maintained in a humidified atmosphere containing $5 \% \mathrm{CO}_{2}$ at $37{ }^{\circ} \mathrm{C}$ and were passaged using standard cell culture techniques.

\section{Total RNA extraction and CDNA synthesis}

Total RNA was extracted using TRIzol reagent (Invitrogen, Carlsbad, CA, USA), according to the manufacturer's protocol. The concentration and purity of RNA were assessed spectrophotometrically at 260 and $280 \mathrm{~nm}$. cDNA was synthesized from total RNA ( $2 \mu \mathrm{g})$ using M-MLV Reverse Transcriptase (Promega, San Luis Obispo, CA, USA), following the manufacturer's instructions.

\section{Quantitative real-time PCR}

Quantitative real-time PCR was performed with the ABI PRISM 7500 Sequence Detection System (Applied Biosystems) using a SYBR Premix DimerEraser kit (Takara Biotechnology, Dalian, Liaoning, China). Amplification reactions included $1 \mu \mathrm{l}$ cDNA template, $0.3 \mu \mathrm{l}$ each of the forward and reverse primers $(10 \mu \mathrm{M}), 0.2 \mu \mathrm{l}$ of $50 \times$ ROX Reference Dye II (Takara), and $5 \mu$ l of $2 \times$ SYBR Premix DimerEraser in a total volume of $10 \mu \mathrm{l}$. The primers used were 5'-AATCTGAGGCGACTGGTGA3' (forward) and 5'-CTCCTCCTGTTCGACAGTCA-3' (reverse) for MFN2 and 5'-CTTAGTTGCGTTACA CCCTTTC-3' (forward) and 5'-CACCTTCACCGTT CCAGTTT-3' (reverse) for $\beta$-actin. The transcripts were amplified with an initial denaturation at $95{ }^{\circ} \mathrm{C}$ for $30 \mathrm{~s}$, followed by 40 cycles at $95{ }^{\circ} \mathrm{C}$ for $5 \mathrm{~s}, 55^{\circ} \mathrm{C}$ for $30 \mathrm{~s}$, and $72{ }^{\circ} \mathrm{C}$ for $34 \mathrm{~s}$. The comparative threshold cycle $\left(2^{-\Delta \Delta \mathrm{CT}}\right)$ method was used for relative quantification. $\beta$-Actin was used as an internal control for normalization. All realtime PCRs were performed in triplicate to evaluate data reproducibility.

\section{Plasmid transfection}

The plasmid vector (pIRES2-EGFP Vector) and plasmidMFN2 were purchased from Invitrogen, USA. The plasmids were transfected in HepG2 cells using Lipofectamine 2000 (Invitrogen), according to the manufacturer's instructions. The efficiency of transfection was evaluated by qPCR and western blot analysis after plasmid treatment for $48 \mathrm{~h}$.

\section{Gene expression microarray}

Total RNA was extracted from HepG2 cells transfected with plasmid vector-NC (pIRES2-EGFPVector) or plasmid-MFN2 for $48 \mathrm{~h}$ using TRIzol (Invitrogen). The Affymetrix Human Genome U133Plus 2.0 Array (Affymetrix, USA) was used for gene expression profiling. Microarray experiments were performed at ShanghaiBio (National Engineering Center for Biochips, Shanghai, China). MAS 5.0 and the "Oligo" package from Bioconductor (http://www.bioconductor.org) were used to normalize the data and annotate probe information.

\section{Data preprocessing and identification of DEGs}

Normalized signal intensity data were imported into BRBArrayTools ver. 4.5 (National Cancer Institute, http:// linus.nci.nih.gov/BRB-ArrayTools.html) for preprocessing. We excluded those genes for which the percentage absent exceeded $50 \%$. We identified DEGs using paired $t$ tests with a random variance model. The nominal significance 
level for each univariate test was $<0.05$. Only genes with a fold change of $\geq 2$ were selected as DEGs.

\section{Construction of the MFN2-related FI network}

In total, 217,249 pairs of FIs were downloaded from Reactome [20] (ver. 2014, http://www.reactome.org). These pairwise relationships are derived from datasets of proteinprotein interactions from BioGRID [21], the Database of Interacting Proteins [22], the Human Protein Reference Database [23], I2D [24], IntACT [25], and MINT [26]. This interaction information was imported into Cytoscape ver. 3.2.1 [27] (http://www.cytoscape.org). By mapping the MFN2-related DEGs to the FI data, we constructed the MFN2-related FI network.

\section{Pathway enrichment analysis for the FI network}

The ReactomeFIViz app was used in Cytoscape for pathway enrichment analysis [28]. The sources of pathway annotations include Cell Map (http://cancer.cellmap.org), Reactome [20], the Kyoto Encyclopedia of Genes and Genomes (KEGG) [29], Panther Pathways [30], NCI-PID [31], and BioCarta (http://www.biocarta.com/genes/index.asp). A false discovery rate (FDR) of $<0.05$ was selected as the cutoff criterion.

\section{Statistical analysis}

Statistical analyses of continuous variables are presented the mean \pm standard deviation. The non-parametric Mann-Whitney $U$ test was used to analyze differences in the MFN2 mRNA expression between the tumor tissue and corresponding non-tumor tissue. Pearson's chisquare test was used to compare categorical variables, whereas Student's $t$ test was used for continuous variables. We divided the patients into high and low expression groups using the median MFN2 mRNA expression as a cutoff because the median is not affected by extreme values (outliers). Survival curves were generated using the Kaplan-Meier method, and the differences were compared using the log-rank test. Multivariate analysis was performed using the Cox proportional hazard regression model. A two-tailed $p$ value of $<0.05$ was considered statistically significant. All statistical analyses were performed using the Statistical Package for the Social Sciences (SPSS 20.0 for Windows, SPSS, Chicago, IL). Graphs were created using GraphPad Prism (ver. 6.01).

\section{Results}

\section{Expression of MFN2 mRNA in HCC paired tissues}

MFN2 mRNA levels were determined in 115 pairs of human HCC and corresponding non-tumor hepatic tissues. The MFN2 mRNA expression was higher in $76.5 \%$ of the non-tumor hepatic tissues than in the paired HCC tissue (Additional file 2: Figure S1A). MFN2 was significantly $(p<$
0.0001) downregulated in tumor tissue compared with non-tumor tissue (Additional file 2: Figure S1B), with average mRNA expression levels of $6.76 \pm 8.04$ and $4.34 \pm 6.06$, respectively (Table 1).

\section{Correlation between MFN2 expression and clinicopathological parameters}

The patients with HCC were divided into low $(n=58)$ and high $(n=57)$ expression groups based on the median value of MFN2 expression. Table 2 lists the patients' clinicopathological variables. Sex and preoperative alpha-fetoprotein correlated significantly $(p<0.05)$ with MFN2 mRNA expression, whereas age, HBsAg, HBV-DNA replication, liver cirrhosis, tumor number, tumor size, vascular invasion, lymph node metastasis, intrahepatic metastasis, liver capsular invasion, differentiation, and TNM stage did not.

\section{Prognostic significance of MFN2 expression}

Evaluating the relationship between MFN2 mRNA expression and patient survival using the Kaplan-Meier survival curve, patients with relatively high MFN2 expression in HCC had substantially longer overall survival (OS) than those with low MFN2 expression $(p<0.05)$ (Fig. 1a). However, MFN2 expression did not seem to affect the recurrence-free survival $(p>0.05)$ (Fig. 1b), even after considering postoperative prophylactic transhepatic arterial chemotherapy and embolization (TACE) (Fig. 1c).

Furthermore, univariate analysis revealed that liver capsule invasion and MFN2 expression were significant predictors of OS (Table 3). Multivariate analysis using the Cox proportional hazards model also indicated that liver capsule invasion (hazard ratio $(\mathrm{HR})=7.206, p=0.011$ ) and MFN2 expression ( $\mathrm{HR}=0.063, p=0.009)$ were independent predictors in patients with $\mathrm{HCC}$ (Table 3).

\section{Identification of DEGs after MFN2 overexpression}

In total, 364 genes were differentially expressed (Additional file 3: Table S2) in HepG2 cells transfected with plasmid-MFN2 compared with HepG2 cells transfected with vector-NC (Additional file 5: Figure S2). Of these, 211 genes $(58 \%)$ were upregulated and the remaining 153 (42\%) were downregulated.

Table 1 Distribution of MFN2 mRNA level and survival in HCC patients

\begin{tabular}{llllll}
\hline Variables & Mean \pm & Range & \multicolumn{3}{l}{ Percentiles } \\
\cline { 4 - 6 } & SD & & 25 & 50 & 75 \\
\hline MFN2 in non-tumor tissues & $6.76 \pm 8.04$ & $0.32-58.06$ & 1.55 & 4.05 & 9.32 \\
MFN2 in tumor tissues & $4.34 \pm 6.06$ & $0.20-30.36$ & 0.99 & 1.81 & 4.66 \\
\hline
\end{tabular}

MFN2 mitofusion 2 
Table 2 Correlation between MFN2 expression in tumor tissue with clinicopathological factors in hepatocellular carcinoma patients

\begin{tabular}{|c|c|c|c|}
\hline \multirow[t]{3}{*}{ Variables } & \multicolumn{2}{|l|}{ MFN2 expression } & \multirow{3}{*}{$P$ value } \\
\hline & \multirow{2}{*}{$\begin{array}{l}\text { Low } \\
n=58\end{array}$} & \multirow{2}{*}{$\begin{array}{l}\text { High } \\
n=57\end{array}$} & \\
\hline & & & \\
\hline Age (year) & $57.1 \pm 11.4$ & $57.1 \pm 9.9$ & 0.996 \\
\hline Gender (female/male) & $3 / 55$ & $12 / 45$ & 0.012 \\
\hline HBsAg (no/yes) & $15 / 43$ & $7 / 50$ & 0.065 \\
\hline $\begin{array}{l}\text { HBV-DNA replication } \\
\text { (no/yes) }\end{array}$ & $34 / 24$ & $32 / 25$ & 0.789 \\
\hline $\begin{array}{l}\text { Liver cirrhosis } \\
\text { (no/yes) }\end{array}$ & $22 / 36$ & $18 / 39$ & 0.476 \\
\hline Preoperative AFP & $6292.3 \pm 14073.3$ & $4075.1 \pm 13793.9$ & 0.048 \\
\hline $\begin{array}{l}\text { Tumor number } \\
(1 />1)\end{array}$ & $44 / 14$ & $42 / 15$ & 0.789 \\
\hline Tumor size $(\mathrm{cm})$ & $6.9 \pm 3.6$ & $6.3 \pm 3.0$ & 0.610 \\
\hline $\begin{array}{l}\text { PV or VI invasion } \\
\text { (no/yes) }\end{array}$ & $37 / 21$ & $43 / 14$ & 0.177 \\
\hline $\begin{array}{l}\text { Lymph node } \\
\text { metastasis (no/yes) }\end{array}$ & $51 / 7$ & $47 / 10$ & 0.410 \\
\hline $\begin{array}{l}\text { Intrahepatic } \\
\text { metastasis (no/yes) }\end{array}$ & $38 / 20$ & $33 / 24$ & 0.402 \\
\hline $\begin{array}{l}\text { Liver capsular } \\
\text { invasion (no/yes) }\end{array}$ & $40 / 18$ & $41 / 16$ & 0.729 \\
\hline TNM stage (I/II-IV) & $23 / 35$ & $22 / 35$ & 0.908 \\
\hline $\begin{array}{l}\text { Differentiation } \\
\text { (well/moderate } \\
\text { or poor) }\end{array}$ & $20 / 38$ & $28 / 29$ & 0.113 \\
\hline
\end{tabular}

$H B s A g$ hepatitis $B$ surface antigen, $H B V-D N A$ hepatitis $B$ virus deoxyribonucleic acid, $A F P$ alpha-fetoprotein, $P V$ portal vein, $V I$ intrahepatic vein

\section{Analysis of FI network affected by MFN2}

By mapping the MFN2-related DEGs to the FI data, we constructed the MFN2-related FI network. This network comprised 93 nodes in 11 clusters, with the largest cluster containing 62 nodes (Fig. 2a). Using hierarchical clustering based on the gene expression level (Fig. 2b), the 93 genes in the network could be differentiated into two groups. The 93 nodes were connected via 114 FIs, corresponding to an effective mean degree of 2.5. Degree refers to the number of nearest neighbors of a node. Nodes with degrees of $\geq 5$ were selected as hub nodes. The hub nodes in the FI network were JUN, JAK2, FN1, MAP2K6, ITGA6, RPS15A, PLCB4, RBM8A, RPS28, IGF1R, TBL1XR1, and SP1, suggesting that these genes are related to MFN2.

To functionally classify these 93 significant genes, we used ReactomeFIViz to identify significant enrichment of these genes in 18 pathways (Fig. 2c, Additional file 4: Table S3). The most significant pathways were the PI3KAkt signaling pathway, cytokine-cytokine receptor interaction, focal adhesion, influenza $\mathrm{A}$, and direct p53 effectors.

\section{Discussion}

Mitofusin 2 was first recognized as a key protein not only regulating mitochondria fusion but also participating in tumor cell proliferation. Although MFN2 has not been proven to act as a tumor suppressor gene in cancer cells [32], its antitumor function, as revealed in various tumors, continues to be accepted. Previously, we proved that overexpression of the MFN2 gene in HCC resulted in tumor cell apoptosis via mitochondrial pathways mediated by calcium influx [14-16]. MFN2 is a downstream target gene of P53 [33], and such direct regulation is altered by hepatitis $B$ virus $X$ protein in HCC [34]. Here, we examined the clinical significance of MFN2 mRNA expression in 115 HCC specimens. MFN2 was downregulated dramatically in HCC tissues, which is consistent with our previous findings. In fact, we have investigated the possible mechanism of MFN2 downregulation for about 8 years. Firstly, we suspected MFN2 gene downregulated by aberrant promoter CpG methylation. However, we found normal methylation level in MFN2 gene promoter region (data not shown here). Secondly, a lot of potential upstream genes of
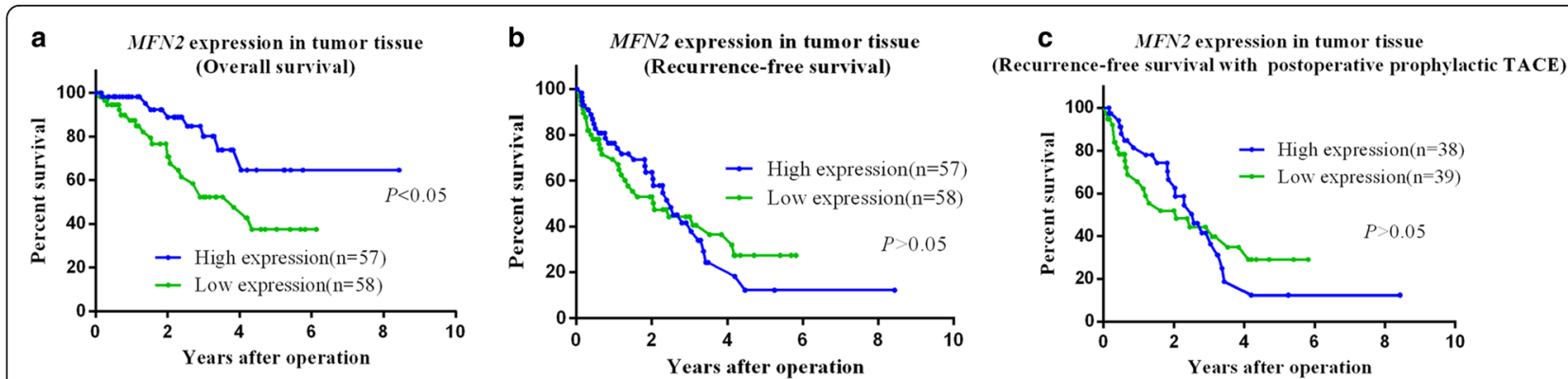

Fig. 1 Survival curves for patients with HCC with high and low MFN2 expression were plotted using the Kaplan-Meier method, and the differences were evaluated using the log-rank test. a MFN2 expression differed significantly with the overall survival rates between the two groups. b, c However, no significant difference was found in the recurrence-free survival rates, even considering postoperative prophylactic TACE 
Table 3 Risk factor analysis of overall survival in tumor tissue

\begin{tabular}{|c|c|c|c|c|}
\hline \multirow[t]{2}{*}{ Prognositic factors } & \multicolumn{2}{|l|}{ Univariate analysis } & \multicolumn{2}{|l|}{ Multivariate analysis } \\
\hline & $\mathrm{HR}(95 \% \mathrm{Cl})$ & $P$ value & $\mathrm{HR}(95 \% \mathrm{Cl})$ & $P$ value \\
\hline Age $(<60 / \geq 60)$ & $0.704(0.250-1.983)$ & 0.507 & & \\
\hline Gender (female/male) & $0.263(0.034-2.007)$ & 0.197 & & \\
\hline HBsAg (no/yes) & $1.688(0.379-7.521)$ & 0.492 & & \\
\hline HBV-DNA replication(no/yes) & $1.491(0.539-4.122)$ & 0.441 & & \\
\hline Liver cirrhosis (no/yes) & $1.117(0.355-3.515)$ & 0.850 & & \\
\hline Preoperative AFP $(<20 / \geq 20 \mathrm{ng} / \mathrm{ml})$ & $0.741(0.262-2.100)$ & 0.573 & & \\
\hline Preoperative AFP $(<400 / \geq 400 \mathrm{ng} / \mathrm{ml})$ & $1.033(0.351-3.035)$ & 0.954 & & \\
\hline Tumor number $(1 />1)$ & $0.861(0.251-3.174)$ & 0.893 & & \\
\hline Tumor size $(<5 / \geq 5 \mathrm{~cm})$ & $1.304(0.462-3.677)$ & 0.616 & & \\
\hline Tumor size $(<8 / \geq 8 \mathrm{~cm})$ & $2.193(0.729-6.594)$ & 0.162 & & \\
\hline PV or VI invasion (no/yes) & $0.959(0.268-3.433)$ & 0.949 & & \\
\hline PVTT (no/yes) & $0.517(0.067-3.973)$ & 0.526 & & \\
\hline Lymph node metastasis (no/yes) & $0.773(0.098-6.077)$ & 0.807 & & \\
\hline Intrahepatic metastasis (no/yes) & $1.264(0.429-3.726)$ & 0.670 & & \\
\hline Liver capsular invasion (no/yes) & $5.811(1.975-17.096)$ & 0.001 & $7.206(1.571-33.063)$ & 0.011 \\
\hline TNM stage (I/II-IV) & $2.322(0.772-6.980)$ & 0.134 & & \\
\hline Differentiation (well/moderate or poor) & $1.529(0.541-4.318)$ & 0.423 & & \\
\hline TACE (no/yes) & $0.877(0.276-2.794)$ & 0.825 & & \\
\hline MFN2 expression (low/high) & $0.263(0.074-0.933)$ & 0.039 & $0.063(0.008-0.496)$ & 0.009 \\
\hline
\end{tabular}

HBs $A g$ hepatitis B surface antigen, $H B V$-DNA hepatitis B virus deoxyribonucleic acid, AFP alpha-fetoprotein, $P V$ portal vein, $V I$ intrahepatic vein, $P V T T$ portal vein tumor thrombus, MFN2 mitofusion 2, TACE transcatheter arterial chemoembolization

MFN2 were chosen to be investigated. Fortunately, we found that MFN2 is a novel target of P53 which may partly explain the low expression level of MFN2 in HCC [33]. Furthermore, MicroRNAs were considered as candidate regulation factors of MFN2. And we did demonstrate an upregulated MicroRNA called miR761 that could directly regulate MFN2 in HCC [35]. We suggest that the possible molecular mechanism behind MFN2 downregulated is very complicated that cannot be clarified by one factor recently. Maybe, a new critical factor served as a MFN2 regulator will be found in the future.

According to statistical analysis in Table 2, we found that tumor MFN2 expression was significantly correlated with sex and the preoperative alpha-fetoprotein level. A recent paper reported significant differences in the expression of a key regulator of mitochondrial biogenesis between males and females in the mouse brain [36]. Therefore, it is possible that MFN2 is expressed differently in human males and females. However, there may be sampling errors in these studies; a larger sample is needed to confirm this finding. Additionally, as a tumor marker, AFP served as an important indicator for HCC diagnosis and patient follow-up. In our findings, preoperative AFP level in serum and MFN2 mRNA level in HCC tissue showed opposite tendency, hypothesized a potential regulation relationships between them. However, we had not found any evidence to demonstrate this hypothesis yet. Combined with survival analysis, we thought the patient who had a high level of MFN2 and low level preoperative AFP may have a better overall survival after operation. But such interpretation was not logical because various factors could affect overall survival rate after hepatectomy for HCC patient. Perhaps, it is better to correlate MFN2 with postoperative AFP. However, we were unable to obtain all postoperative AFP level in this study.

Despite the weak relationships between MFN2 expression and clinicopathological parameters, survival analysis showed that a higher MFN2 expression level was associated with better postoperative survival of patients with HCC. Furthermore, univariate analysis revealed that liver capsule invasion and MFN2 expression were significant predictors of OS. Therefore, MFN2 has an important role in the development of HCC. We propose that MFN2 could serve as a biomarker in HCC tissue for predicting survival after hepatectomy.

Most research of MFN2 in tumors has concentrated on the regulation of mitochondrial function. For a more comprehensive evaluation of MFN2-associated genes, we used a comparative gene expression microarray. This microarray 


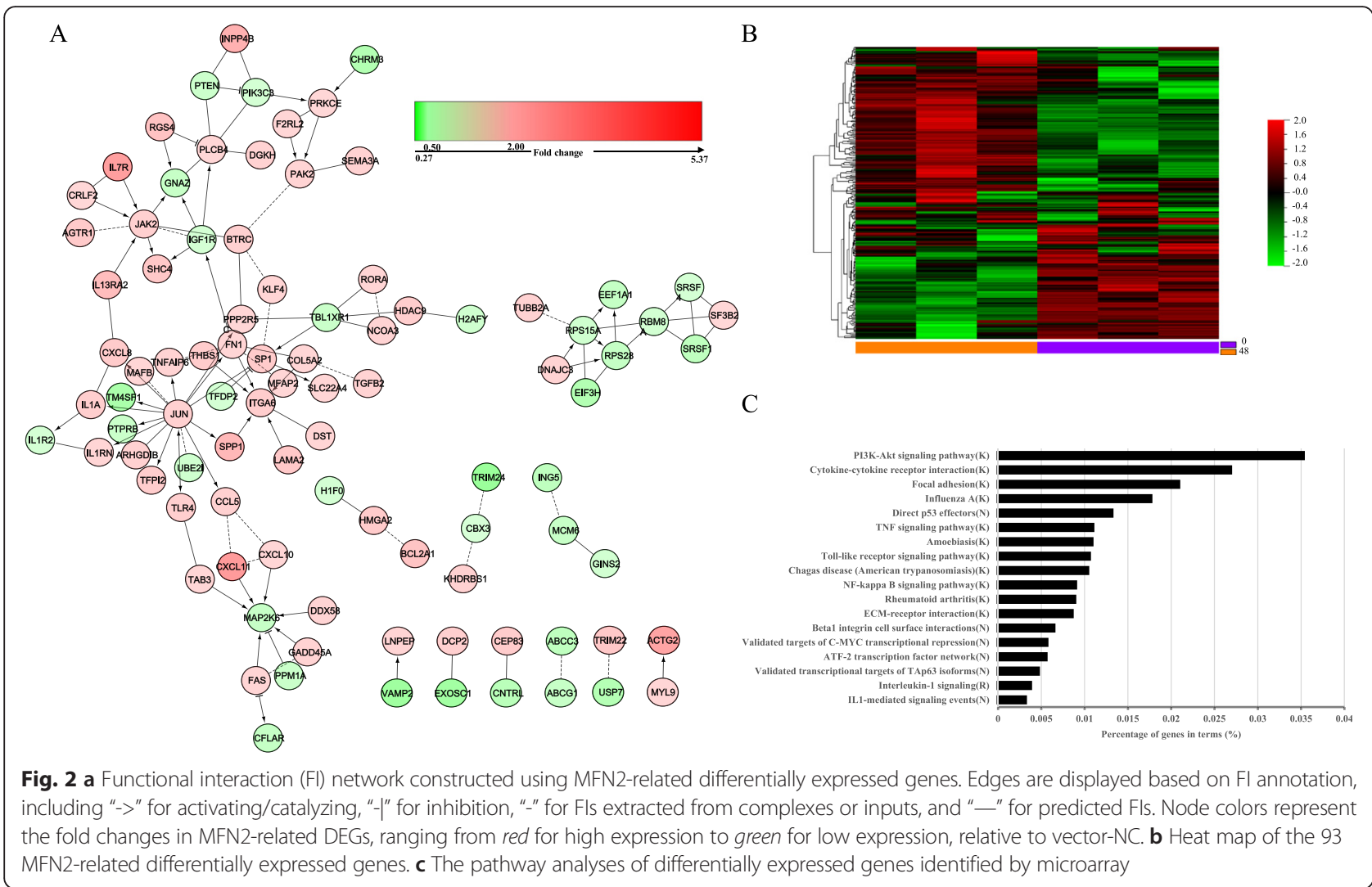

revealed 364 DEGs after MFN2 overexpression, of which 93 significant genes were classified functionally into 18 pathways, including the PI3K-Akt signaling pathway, cytokine-cytokine receptor interaction, focal adhesion, and direct p53 effectors. As we know, these pathways play important roles in tumor development and progression [37-40]. Multiple genes, such as mTOR, NF-kB, BCL2, and BAX, participate in PI3K-AKT signal pathway in HCC [4143]. Therefore, MFN2 may function powerfully through regulating PI3K-AKT. For cytokine-cytokine receptor interaction and focal adhesion, it makes MFN2 possible to regulate such membrane receptors result in tumor cell migration and invasion which will bring us new sight about how MFN2 inhibits tumor cell metastasis. Previous research has found that MFN2 was related to virus infection mechanism [44] which could verify our findings with respect to influenza A. It is interesting that the relationship between MFN2 and P53 may create a positive feedback mechanism based on our findings [33]. These results support previous studies of MFN2 in HCC and may shed new light on the complicated proapoptotic and antiproliferative mechanism of MFN2 in the tumor.

In the future, our first-step study on MFN2 may focus on verifying the comparative microarray analysis results by various experimental methods. After that, we will select a promising downstream pathway of MFN2 to further investigate the regulation mechanism. A cell membrane receptor regulated by MFN2 is also taken into our account in our future research.

\section{Conclusions}

In conclusion, our data suggest that the MFN2 expression level in tumors is closely related to the survival of patients with HCC after hepatectomy. Numerous critical signaling pathways take part in the MFN2-mediated functional changes in HCC.

\section{Additional files}

Additional file 1: Table S1: Patients demographic and clinicopathological characteristics. (DOC $52 \mathrm{~kb}$ )

Additional file 2: Figure S1: Expression of MFN2 mRNA in HCC tumors and corresponding non-tumor hepatic tissues. (A) MFN2 was downregulated in most HCC tumors (76.5\%). (B) MFN2 was significantly ( $p<$ 0.0001) downregulated in the tumor compared with the non-tumor hepatic counterpart using the non-parametric Mann-Whitney $U$ test. Standard error of the mean (SEM) was used as error bar. (TIF $59 \mathrm{~kb}$ )

Additional file 3: Table S2: DEGs after MFN2 overexpression in HepG2 cells. (DOC $197 \mathrm{~kb}$ )

Additional file 4: Table S3: Significant enrichment of 93 genes in 18 pathways. (DOC $47 \mathrm{~kb}$ )

Additional file 5: Figure S2: Efficiency of transfection evaluation by $\mathrm{qPCR}$ and western blot analysis in HepG2 cells. (A) MFN2 mRNA was significantly upregulated by plasmid-MFN2. Standard deviation (SD) was used as error bar. (B) MFN2 protein was also upregulated by plasmid-MFN2. (TIF $46 \mathrm{~kb}$ ) 


\section{Abbreviations}

$C T$, computed tomography; DEGs, differentially expressed genes; DMEM, Dulbecco's modified Eagle's medium; Fl, functional interaction; HCC, hepatocellular carcinoma; KEGG, Kyoto Encyclopedia of Genes and Genomes; MFN2, mitofusin-2; MRI, magnetic resonance imaging; OS, overall survival

\section{Acknowledgements}

This manuscript has been checked and proofread by two professional editors from Textcheck.

\section{Funding}

This work was funded by projects 81172315 and 81572307 supported by National Natural Science Foundation of China, Natural Science Foundation of Zhejiang Province (LY14H160022) and the Major Program of Science and Technology of Zhejiang Province (2014C13G2010059).

\section{Availability of data and materials}

The datasets supporting the conclusions of this article are available in the repository of Ethics Committee for Clinical Research of First Affiliated Hospital of Zhejiang University.

\section{Authors' contributions}

YSW and DKZ designed the research and wrote the manuscript (They contributed equally to this work). XBX, WS, XHZ, and PFH did the experiments and collected the data. XYZ and HG analyzed the data and performed the statistical analysis. SSZ and WLW reviewed and edited the manuscript. All authors read and approved the final manuscript.

\section{Competing interests}

The authors declare that they have no competing interests.

\section{Consent for publication}

A copy of the written consent is available for review by the Editor-in-Chief of this journal.

\section{Ethics approval and consent to participate}

The study was reviewed and approved by the Zhejiang University Institutional Review Board. And all study participants, or their legal guardian, provided informed written consent prior to study enrollment.

\section{Author details}

'Division of Hepatobiliary and Pancreatic Surgery, Department of Surgery, First Affiliated Hospital, School of Medicine, Zhejiang University, No. 79 Qingchun Road, Hangzhou 310003, China. ${ }^{2}$ Key Lab of Combined Multi-organ Transplantation, Ministry of Public Health, No. 79 Qingchun Road, Hangzhou 310003, China. ${ }^{3}$ Collaborative Innovation Center for Diagnosis and Treatment of Infectious Diseases, No. 79 Qingchun Road, Hangzhou 310003, China.

Received: 23 December 2015 Accepted: 15 June 2016 Published online: 07 July 2016

\section{References}

1. El-Serag HB. Hepatocellular carcinoma. N Engl J Med. 2011:365:1118-27.

2. Ferlay J, Shin HR, Bray F, Forman D, Mathers C, Parkin DM. Estimates of worldwide burden of cancer in 2008: Globocan 2008. Int J Cancer. 2010;127:2893-917.

3. European Association for Study of $L$, European Organisation for $R$ Treatment of C. EASL-EORTC clinical practice guidelines: management of hepatocellular carcinoma. Eur J Cancer. 2012;48:599-641.

4. Land $H$, Parada LF, Weinberg RA. Cellular oncogenes and multistep carcinogenesis. Science. 1983;222:771-8.

5. Chiba T, Suzuki E, Saito T, Ogasawara S, Ooka Y, Tawada A, et al. Biological features and biomarkers in hepatocellular carcinoma. World J Hepatol. 2015:7:2020-8

6. Bourne HR, Sanders DA, McCormick F. The GTPase superfamily: a conserved switch for diverse cell functions. Nature. 1990;348:125-32.

7. Rojo M, Legros F, Chateau D, Lombes A. Membrane topology and mitochondrial targeting of mitofusins, ubiquitous mammalian homologs of the transmembrane gtpase fzo. J Cell Sci. 2002;115:1663-74.
8. Ding Y, Gao H, Zhao L, Wang X, Zheng M. Mitofusin 2-deficiency suppresses cell proliferation through disturbance of autophagy. PLoS One. 2015;10:e0121328

9. Zuchner S, Mersiyanova IV, Muglia M, Bissar-Tadmouri N, Rochelle J, Dadali EL, et al. Mutations in the mitochondrial GTPase mitofusin 2 cause CharcotMarie-Tooth neuropathy type 2A. Nat Genet. 2004;36:449-51.

10. Bach D, Pich S, Soriano FX, Vega N, Baumgartner B, Oriola J, et al. Mitofusin2 determines mitochondrial network architecture and mitochondrial metabolism. A novel regulatory mechanism altered in obesity. J Biol Chem. 2003;278:17190-7.

11. Hernandez-Alvarez MI, Thabit H, Burns N, Shah S, Brema I, Hatunic M, et al. Subjects with early-onset type 2 diabetes show defective activation of the skeletal muscle PGC-1\{alpha\}/mitofusin-2 regulatory pathway in response to physical activity. Diabetes Care. 2010;33:645-51.

12. Guo X, Chen KH, Guo Y, Liao H, Tang J, Xiao RP. Mitofusin 2 triggers vascular smooth muscle cell apoptosis via mitochondrial death pathway. Circ Res. 2007:101:1113-22

13. Shen T, Zheng M, Cao C, Chen C, Tang J, Zhang W, et al. Mitofusin-2 is a major determinant of oxidative stress-mediated heart muscle cell apoptosis. J Biol Chem. 2007;282:23354-61.

14. Wang W, Zhu F, Wang S, Wei J, Jia C, Zhang Y, et al. HSG provides antitumor efficacy on hepatocellular carcinoma both in vitro and in vivo. Oncol Rep. 2010;24:183-8.

15. Wang W, Lu J, Zhu F, Wei J, Jia C, Zhang Y, et al. Pro-apoptotic and antiproliferative effects of mitofusin-2 via bax signaling in hepatocellular carcinoma cells. Med Oncol. 2012;29:70-6.

16. Wang W, Xie Q, Zhou X, Yao J, Zhu X, Huang P, et al. Mitofusin-2 triggers mitochondria $\mathrm{Ca} 2+$ influx from the endoplasmic reticulum to induce apoptosis in hepatocellular carcinoma cells. Cancer Lett. 2015;358:47-58.

17. Wu L, Li Z, Zhang Y, Zhang P, Zhu X, Huang J, et al. Adenovirus-expressed human hyperplasia suppressor gene induces apoptosis in cancer cells. Mol Cancer Ther. 2008;7:222-32.

18. Jin B, Fu G, Pan H, Cheng X, Zhou L, Lv J, et al. Anti-tumour efficacy of mitofusin2 in urinary bladder carcinoma. Med Oncol. 2011;28 Suppl 1:S373-80.

19. Zhang GE, Jin HL, Lin XK, Chen C, Liu XS, Zhang Q, et al. Anti-tumor effects of Mfn2 in gastric cancer. Int J Mol Sci. 2013;14:13005-21.

20. Croft D, O'Kelly G, Wu G, Haw R, Gillespie M, Matthews L, et al. Reactome: a database of reactions, pathways and biological processes. Nucleic Acids Res. 2011;39:D691-7.

21. Chatr-Aryamontri A, Breitkreutz BJ, Oughtred R, Boucher L, Heinicke S, Chen D, et al. The biogrid interaction database: 2015 update. Nucleic Acids Res. 2015;43:D470-8.

22. Salwinski L, Miller CS, Smith AJ, Pettit FK, Bowie JU, Eisenberg D. The database of interacting proteins: 2004 update. Nucleic Acids Res. 2004:32:D449-51.

23. Keshava Prasad TS, Goel R, Kandasamy K, Keerthikumar S, Kumar S, Mathivanan S, et al. Human protein reference database_-2009 update. Nucleic Acids Res. 2009;37:D767-72

24. Brown KR, Jurisica I. Unequal evolutionary conservation of human protein interactions in interologous networks. Genome Biol. 2007;8:R95.

25. Orchard S, Ammari M, Aranda B, Breuza L, Briganti L, Broackes-Carter F, et al The MlntAct project-intact as a common curation platform for 11 molecular interaction databases. Nucleic Acids Res. 2014:42:D358-63.

26. Licata L, Briganti L, Peluso D, Perfetto L, lannuccelli M, Galeota E, et al. MINT, the molecular interaction database: 2012 update. Nucleic Acids Res. 2012:40:D857-61.

27. Shannon P, Markiel A, Ozier O, Baliga NS, Wang JT, Ramage D, et al. Cytoscape: a software environment for integrated models of biomolecular interaction networks. Genome Res. 2003;13:2498-504.

28. Wu G, Dawson E, Duong A, Haw R, Stein L. Reactomefiviz: a cytoscape app for pathway and network-based data analysis. F1000Res. 2014;3:146.

29. Kanehisa M. The KEGG database. Novartis Found Symp. 2002;247:91-101. discussion -3, 19-28, 244-52.

30. Mi H, Muruganujan A, Thomas PD. PANTHER in 2013: modeling the evolution of gene function, and other gene attributes, in the context of phylogenetic trees. Nucleic Acids Res. 2013;41:D377-86.

31. Schaefer CF, Anthony K, Krupa S, Buchoff J, Day M, Hannay T, et al. PID: the pathway interaction database. Nucleic Acids Res. 2009;37:D674-9.

32. Lou Y, Li R, Liu J, Zhang Y, Zhang X, Jin B, et al. Mitofusin-2 over-expresses and leads to dysregulation of cell cycle and cell invasion in lung adenocarcinoma. Med Oncol. 2015;32:132. 
33. Wang W, Cheng X, Lu J, Wei J, Fu G, Zhu F, et al. Mitofusin-2 is a novel direct target of p53. Biochem Biophys Res Commun. 2010;400:587-92.

34. Wang W, Zhou D, Wei J, Wu Z, Cheng X, Sun Q, et al. Hepatitis b virus $x$ protein inhibits p53-mediated upregulation of mitofusin-2 in hepatocellular carcinoma cells. Biochem Biophys Res Commun. 2012:421:355-60

35. Wang W, Lin H, Zhou L, Zhu Q, Gao S, Xie H, et al. Microrna-30a-3p inhibits tumor proliferation, invasiveness and metastasis and is downregulated in hepatocellular carcinoma. Eur J Surg Oncol. 2014;40:1586-94.

36. Zawada I, Masternak MM, List EO, Stout MB, Berryman DE, Lewinski A, et al. Gene expression of key regulators of mitochondrial biogenesis is sex dependent in mice with growth hormone receptor deletion in liver. Aging. 2015;7:195-204

37. Bunney TD, Katan M. Phosphoinositide signalling in cancer: beyond pi3k and pten. Nat Rev Cancer. 2010:10:342-52.

38. Kalvakolanu DV, Nallar SC, Kalakonda S. Cytokine-induced tumor suppressors: a grim story. Cytokine. 2010;52:128-42.

39. Chen JS, Huang XH, Wang Q, Chen XL, Fu XH, Tan HX, et al. FAK is involved in invasion and metastasis of hepatocellular carcinoma. Clin Exp Metastasis. 2010;27:71-82.

40. Schmitt CA, Fridman JS, Yang M, Baranov E, Hoffman RM, Lowe SW. Dissecting p53 tumor suppressor functions in vivo. Cancer Cell. 2002;1:289-98.

41. Sui Y, Zheng X, Zhao D. Rab31 promoted hepatocellular carcinoma (hcc) progression via inhibition of cell apoptosis induced by pi3k/akt/bcl-2/bax pathway. Tumour Biol. 2015;36:8661-70.

42. Cui SX, Shi WN, Song ZY, Wang SQ Yu XF, Gao ZH, et al. Des-gammacarboxy prothrombin antagonizes the effects of Sorafenib on human hepatocellular carcinoma through activation of the Raf/MEK/ERK and PIBK Akt/mTOR signaling pathways. Oncotarget. 2016. Epub ahead of print

43. Qiu FN, Huang Y, Chen DY, Li F, Wu YA, Wu WB, et al. Eukaryotic elongation factor-1alpha 2 knockdown inhibits hepatocarcinogenesis by suppressing PI3K/Akt/NF-kappaB signaling. World J Gastroenterol. 2016;22:4226-37.

44. Ichinohe T, Yamazaki T, Koshiba T, Yanagi Y. Mitochondrial protein mitofusin 2 is required for NLRP3 inflammasome activation after RNA virus infection. Proc Natl Acad Sci U S A. 2013;110:17963-8.

\section{Submit your next manuscript to BioMed Central and we will help you at every step:}

- We accept pre-submission inquiries

- Our selector tool helps you to find the most relevant journal

- We provide round the clock customer support

- Convenient online submission

- Thorough peer review

- Inclusion in PubMed and all major indexing services

- Maximum visibility for your research

Submit your manuscript at www.biomedcentral.com/submit

) Biomed Central 